\title{
Melioidosis parotitis in children
}

\author{
David Dance ${ }^{1,2,3}$ (iD
}

\begin{abstract}
A recent paper published in JVATiTD reporting a child in Hainan with parotitis caused by Burkholderia pseudomallei misleadingly described parotitis as a rare manifestation of melioidosis. In fact, it is one of the commonest forms of paediatric melioidosis seen in other parts of Southeast Asia, although interestingly not in Australia.
\end{abstract}

Keywords: Melioidosis, Parotitis, B. pseudomallei, Paediatric, Children

To: The Editor, Journal of Venomous Animals and Toxins including Tropical Diseases

Sir,

Melioidosis (Burkholderia pseudomallei infection) is an important public health problem in some parts of the world. The incidence has probably been grossly underestimated in the past [1], so it is encouraging that reports have recently begun to emerge from several countries across the tropics, including Hainan island in China [2-4]. The most recent such report, published in your journal, related to a case of parotitis in a child [5]. Unfortunately, this report contained a highly misleading statement about melioidosis parotitis, namely that it was rare and had only been reported following systemic melioidosis. In fact it has been known for many years that parotitis is one of the commonest manifestations of melioidosis in children in SE Asia [6, 7], although interestingly not in Australia [8]. This should have been revealed by a simple literature search using the terms 'melioidosis' and 'parotitis' - a PubMed search using these terms on 14th November 2016 yielded 16 'hits' including the references cited above.

Whilst I am sure that this was a genuine mistake, I think it is important that this misleading impression is corrected.

Yours sincerely

Prof. David Dance, MB, ChB, MSc, FRCPath

\section{Author details}

${ }^{1}$ Senior Clinical Research Fellow/Consultant Microbiologist, Lao-Oxford-Mahosot Hospital-Wellcome Trust Research Unit (LOMWRU), Department of Microbiology, Mahosot Hospital, Vientiane, Lao PDR. ${ }^{2}$ Centre for Tropical Medicine \& Global Health, Nuffield Department of Clinical Medicine, University of Oxford, Oxford, England, UK. ${ }^{3}$ Honorary Professor, Faculty of Infectious and Tropical Diseases, London School of Hygiene and Tropical Medicine, London, England, UK.

Received: 27 November 2016 Accepted: 30 November 2016 Published online: 05 December 2016

\section{References}

1. Limmathurotsakul D, Golding N, Dance DAB, Messina JP, Pigott DM, Moyes CL, et al. Predicted global distribution of Burkholderia pseudomallei and burden of melioidosis. Nat Microbiol. 2016;1:15008.

2. Fang Y, Chen H, Li YL, Li Q, Ye ZJ, Mao XH. Melioidosis in Hainan, China: a restrospective study. Trans R Soc Trop Med Hyg. 2015;109(10):636-42.

3. Fang $Y$, Chen $\mathrm{H}$, Zhu X, Mao X. Fatal melioidosis in a newborn from Hainan, China. Am J Trop Med Hyg. 2016;95(2):444-6.

4. Wang XM, Zheng X, Wu H, Zhou XJ, Kuang HH, Guo HL, et al. Multilocus sequence typing of clinical isolates of Burkholderia pseudomallei collected in Hainan, a tropical island of southern China. Am J Trop Med Hyg. 2016;95(4):760-4.

5. Fu Z, Lin Y, Wu Q, Xia Q. Pediatric suppurative parotitis caused by Burkholderia pseudomallei. J Venom Anim Toxins incl Trop Dis. 2016;22:31.

6. Dance DAB, Davis TM, Wattanagoon Y, Chaowagul W, Saiphan P, Looareesuwan S, et al. Acute suppurative parotitis caused by Pseudomonas pseudomallei in children. J Infect Dis. 1989;159(4):654-60.

7. Stoesser N, Pocock J, Moore CE, Soeng S, Chhat HP, Sar P, et al. Pediatric suppurative parotitis in Cambodia between 2007 and 2011. Pediatr Infect Dis J. 2012;31(8):865-8.

8. Edmond KM, Bauert P, Currie BJ. Paediatric melioidosis in the northern territory of Australia: an expanding clinical spectrum. J Paediatr Child Health. 2001;37(4):337-41.

\footnotetext{
Correspondence: David.d@tropmedres.ac

${ }^{1}$ Senior Clinical Research Fellow/Consultant Microbiologist,

Lao-Oxford-Mahosot Hospital-Wellcome Trust Research Unit (LOMWRU),

Department of Microbiology, Mahosot Hospital, Vientiane, Lao PDR

${ }^{2}$ Centre for Tropical Medicine \& Global Health, Nuffield Department of

Clinical Medicine, University of Oxford, Oxford, England, UK

Full list of author information is available at the end of the article
} 